\title{
NUMERICAL RANGES AND COMPRESSIONS OF $S_{n}$-MATRICES
}

\author{
HWA-LONG GAU AND PEI YuAN Wu
}

Abstract. Let $A$ be an $n$-by- $n(n \geqslant 2) S_{n}$-matrix, that is, $A$ is a contraction with eigenvalues in the open unit disc and with $\operatorname{rank}\left(I_{n}-A^{*} A\right)=1$, and let $W(A)$ denote its numerical range. We show that (1) if $B$ is a $k$-by- $k(1 \leqslant k<n)$ compression of $A$, then $W(B) \varsubsetneqq W(A),(2)$ if $A$ is in the standard upper-triangular form and $B$ is a $k$-by- $k(1 \leqslant k<n)$ principal submatrix of $A$, then $\partial W(B) \cap \partial W(A)=\emptyset$, and (3) the maximum value of $k$ for which there is a $k$-by- $k$ compression of $A$ with all its diagonal entries in $\partial W(A)$ is equal to 2 if $n=2$, and $\lceil n / 2\rceil$ if $n \geqslant 3$.

Mathematics subject classification (2010): 15A60.

Keywords and phrases: Numerical range, compression, $S_{n}$-matrix, unitary dilation.

\section{REFERENCES}

[1] H. Bercovici, Numerical ranges of operators of class $C_{0}$, Linear Multilinear Algebra 50 (2002), 219-222.

[2] I. Chalendar, P. Gorkin And J. R. Partington, Numerical ranges of restricted shifts and unitary dilations, Oper. Matrices 3 (2009), 271-281.

[3] U. DAEPP, P. Gorkin AND R. Mortini, Ellipses and finite Blaschke products, Amer. Math. Monthly 109 (2002), 785-795.

[4] U. Daepp, P. Gorkin And K. Voss, Poncelet's theorem, Sendov's conjecture, and Blaschke products, J. Math. Anal. Appl. 365 (2010), 93-102.

[5] M. R. Embry, The numerical range of an operator, Pacific J. Math. 32 (1970), 647-650.

[6] H.-L. GAU AND P. Y. Wu, Numerical range of $S(\phi)$, Linear Multilinear Algebra 45 (1998), 49-73.

[7] H.-L. Gau And P. Y. WU, Dilation to inflations of $S(\phi)$, Linear Multilinear Algebra 45 (1998), $109-123$.

[8] H.-L. GAU AND P. Y. WU, Lucas' theorem refined, Linear Multilinear Algebra 45 (1999), 359-373.

[9] H.-L. Gau AND P. Y. Wu, Numerical range and Poncelet property, Taiwanese J. Math. 7 (2003), 173-193.

[10] H.-L. GAU AND P. Y. WU, Numerical range circumscribed by two polygons, Linear Algebra Appl. 382 (2004), 155-170.

[11] H.-L. GAU AND P. Y. WU, Numerical range of a normal compression, Linear Multilinear Algebra 52 (2004), 195-201.

[12] H.-L. GAU AND P. Y. WU, Numerical range of a normal compression II, Linear Algebra Appl. 390 (2004), 121-136.

[13] U. HaAgerup AND P. DE LA HARPE, The numerical radius of a nilpotent operator on a Hilbert space, Proc. Amer. Math. Soc. 115 (1992), 371-379.

[14] R. A. Horn and C. R. Johnson, Topics in Matrix Analysis, Cambridge Univ. Press, Cambridge, 1991.

[15] B. Mirman, Numerical ranges and Poncelet curves, Linear Algebra Appl. 281 (1998), 59-85.

[16] B. Mirman, UB-matrices and conditions for Poncelet polygon to be closed, Linear Algebra Appl. 360 (2003), 123-150.

[17] B. Mirman, Poncelet's porism in the finite real plane, Linear Multilinear Algebra 57 (2009), 439 458.

[18] B. Mirman, V. Borovikov, L. Ladyzhensky and R. Vinograd, Numerical ranges, Poncelet curves, invariant measures, Linear Algebra Appl. 329 (2001), 61-75. 
[19] B. Mirman And P. ShUKLA, A characterization of complex plane Poncelet curves, Linear Algebra Appl. 408 (2005), 86-119.

[20] D. SARAson, Generalized interpolation in $H^{\infty}$, Trans. Amer. Math. Soc. 127 (1967), 179-203.

[21] P. Y. WU, Polygons and numerical ranges, Amer. Math. Monthly 107 (2000), 528-540.

[22] T. Yoshino, Introduction to Operator Theory, Longman, Harlow, Essex, 1993.

[23] H.-Y. Zhang, Y.-N. Dou, M.-F. WANG AND H.-K. Du, On the boundary of numerical ranges of operators, Appl. Math. Lett. 24 (2011), 620-622. 